Mycologia, 103(6), 2011, pp. 1184-1193. DOI: 10.3852/11-027

(c) 2011 by The Mycological Society of America, Lawrence, KS 66044-8897

\title{
ITS-1 versus ITS-2 pyrosequencing: a comparison of fungal populations in truffle grounds
}

\author{
Antonietta Mello ${ }^{1,2}$ \\ Istituto per la Protezione delle Piante del CNR, Sezione \\ di Torino, Viale Mattioli 25, 10125 Torino, Italy \\ Chiara Napoli ${ }^{2}$ \\ Dipartimento di Biologia Vegetale dell'Università di \\ Torino, Viale Mattioli 25, 10125 Torino, Italy \\ Claude Murat \\ Emmanuelle Morin \\ INRA, UMR 1136, INRA-Nancy Université, \\ Interactions Arbres/Microorganismes, 54280 \\ Champenoux, France \\ Giuseppe Marceddu \\ Dipartimento di Biologia Vegetale dell'Università di \\ Torino, Viale Mattioli 25, 10125 Torino, Italy \\ Paola Bonfante \\ Dipartimento di Biologia Vegetale dell'Università di \\ Torino, Viale Mattioli 25, 10125 Torino, Italy
}

\begin{abstract}
In a recent study pyrosequencing of the ribosomal internal transcribed spacer-1 (ITS-1) has validated the effectiveness of such technology in the survey of soil fungal diversity. Here we compare the two ITS regions, ITS-1 and ITS-2, of the fungal populations occurring in Tuber melanosporum/Quercus pubescens truffle grounds and sampled in two areas, one devoid of vegetation ("burned", brulé in French) where T. melanosporum fruiting bodies are usually collected, and outside the brulé. TS1F/ITS2 and ITS3/ITS4 were used respectively for the amplification of the ITS-1 and ITS-2 regions. Two amplicon libraries were built, one for inside and the other for outside. A set of 15.788 reads was obtained. After the removal of low quality sequences, 3568 and 3156 sequences were obtained from inside the brulé with the ITS-1 and ITS-2 primers respectively. The sequences obtained from outside the brulé were 4490 with the ITS-1 primers and 2432 with the ITS-2 primers. Most of the sequences obtained for both ITS fragments could be attributed to fungal organisms.

The pair of primers, ITS1-F/ITS2, was more selective, producing fewer non-fungal sequences ( $1 \%$ inside, $3 \%$ outside), in addition to a higher number of sequences, than the pair ITS3/ITS4 (6\% inside, $11 \%$ outside). Although differences are
\end{abstract}

Submitted 20 Jan 2011; accepted for publication 19 Apr 2011.

${ }^{1}$ Corresponding author. E-mail: a.mello@ipp.cnr.it

${ }^{2}$ Authors contributed equally to this work. present in the taxa percentages between ITS-1 and ITS-2, both reveal that Ascomycota were the dominant fungal phylum and that their number decreased moving from inside the brule to outside, while the number of Basidiomycota increased. Taken together, both the short ITS-1 and ITS-2 reads obtained by the high throughput 454 sequencing provide adequate information for taxon assignment and are suitable to correlate the dynamics of the fungal populations to specific environments.

Key words: Pezizales, Tuber melanosporum

\section{INTRODUCTION}

Over the past $20 \mathrm{y}$ the internal trancribed spacer (ITS) of the ribosomal DNA has provided much of what is known about ectomycorrhizal taxonomy, diagnostics and ecology (Horton and Bruns 2001, Mello et al. 2006, Lilleskov and Parrent 2007, Reich et al. 2009). Large scale Sanger sequencing of this region has allowed the characterization of fungal diversity in temperate forest soils and has provided insights into the composition of soil fungal communities (O'Brien et al. 2005). Pyrosequencing of the ITS-1 has validated the effectiveness of such technology, which bypass DNA cloning procedures and produces many more sequences more rapidly for the survey of soil fungal diversity (Buée et al. 2009). This technology, which initially was employed to detect single nucleotide polymorphisms, has been applied successfully in large scale DNA sequencing in multidisciplinary fields with important applications for genome sequencing and metagenomics (Ronaghi et al. 1998, Ronaghi 2001). The first comprehensive examination of soil community diversity was applied to bacteria in four soils across the western hemisphere (Roesch et al. 2007) and then to forest fungi (Buée et al. 2009). Among these fungi an unexpectedly high fungal diversity was found, with the majority of species retrieved only rarely. Because this work relies only on the short ITS-1 reads no information is available on how ITS-2 performs in pyrosequencing.

Therefore the aim of our work was to compare the utility of the two ITS regions, ITS-1 and ITS-2, for assessing diversity of the fungal populations occurring in Tuber melanosporum/Quercus pubescens truffle grounds. We sampled two selected environments, inside and outside a "burned" area, between which marked differences in fungal populations were shown 
by Napoli et al. (2010). The "burned" area (commonly referred to by the French word brule) is soil devoid of vegetation near or around the host tree of the ectomycorrhizal fungus $T$. melanosporum, whose fruit bodies are known as black truffle. This is where these fruiting bodies are usually collected in late autumn and winter (Suz et al. 2008). Napoli et al. (2010) showed that the fungal populations present inside and outside nine brulé areas at Cahors, France, clustered into two groups based on comparison of their denaturing gradient gel electrophoresis (DGGE) profiles: one from the samples inside the brule, and the second one from samples outside. Because the fungal populations of all T. melanosporum $/ Q$. pubescens truffle grounds presented similar patterns one of them was selected for a deeper investigation (i.e. molecular cloning of the entire ITS region). From 192 clones, 96 from inside and 96 outside the brulé, Napoli et al. (2010) demonstrated a dominance of $T$. melanosporum and a reduction of the Basidiomycota ectomycorrhizal fungi within the brulé.

In the present work we wanted to determine whether both the short ITS-1 and ITS-2 reads generated by pyrosequencing could provide adequate information for taxon assignment and produce results similar to those we have obtained for truffle ground fungal populations through the more timeconsuming and expensive molecular cloning of the full ITS region. To compare these two methods we took advantage of the extensive sampling undertaken for DGGE to validate pyrosequencing. ITS1F/ITS2 and ITS3/ITS4 were used respectively for the amplification of the ITS-1 and ITS-2 regions. Biases introduced by these ITS primers also are discussed.

\section{MATERIALS AND METHODS}

Soil sampling and DNA extraction.-The sampling sites are T. melanosporum truffle grounds in Cahors (France), which belong to La Station de la trufficulture de CahorsLe Montat (Station d'expérimentation sur la truffe, Lycée professionnel agricole Lacoste, Le Montat, France). All the truffle grounds are productive with a yield of 100-1000 g (Sourzat pers comm). One well defined brulé was selected for each truffle ground on the basis of its features (e.g. the almost complete absence of vegetation). Soil samples were collected in May 2006 from the nine T. melanosporum $/ Q$. pubescens truffle grounds already investigated by Napoli et al. (2010). Samples of about $200 \mathrm{~g}$ soil were collected inside and outside each brulé at a depth of approximately 10$15 \mathrm{~cm}$ and stored at $-80 \mathrm{C}$ until they were analyzed. The number of samples (generally 2-3) collected for each area was different because of the heterogeneity (i.e. having different shapes and sizes) of the brule and the distance of the brulé limit from a tree trunk. After removing any visible roots or small pebble the soil samples (about $50 \mathrm{~g}$ ) from each area were mixed to make them homogeneous. Two pools (inside and outside the brulé) per truffle ground were created, for a total of 18 pools (nine inside the brulé and nine outside).

The total DNA was extracted from $0.5 \mathrm{~g}$ soil with a Fast DNA Spin Kit for Soil (QIAGEN), with modifications according to Luis et al. (2004). Two extractions were made for each pool and mixed to obtain the most representative DNA of the soil sample. In this way 18 DNA samples were obtained. All these DNA samples were quantified with NanoDrop ${ }^{\mathrm{TM}}$ and concentrations of $20 \mathrm{ng} / \mu \mathrm{L}$ were used in the subsequent PCR procedures.

PCR with fusion primers and purification.-The two pair of primers ITS1F (5'-CTTGGTCATTTAGAGGAAGTAA-3')/ ITS2 (5'-GCTGCGTTCTTCATCGATGC-3') and ITS3 (5'GCATCGATGAAGAACGCAGC-3') /ITS4 (5'-TCCTCCGCTTATTGATATGC-3') were chosen respectively for the amplification of the ITS-1 and the ITS-2 from 18 DNA samples. Because the DNA-sample preparation for amplicon sequencing consists of a PCR amplification reaction with special fusion primers these were generated by addition of primer A (5'-GCCTCCCTCGCGCCATCAG-3') and primer B (5'-GCCTTGCCAGCCCGCTCAG-3') sequences to the $5^{\prime}$ ends of the template-specific primers ITS1F/ITS2 and ITS3/ITS4.

The PCR conditions were: denaturation at $94 \mathrm{C}$ for $4 \mathrm{~min}$, followed by two cycles $92 \mathrm{C}, 55 \mathrm{C}, 68 \mathrm{C}$; two cycles $92 \mathrm{C}, 53 \mathrm{C}$, $68 \mathrm{C}$; two cycles $92 \mathrm{C}, 51 \mathrm{C}, 68 \mathrm{C}$; two cycles $92 \mathrm{C}, 49 \mathrm{C}, 68 \mathrm{C}$; and 29 cycles 92 C, 47 C, 68 C. The reaction was followed by a final extension of $68 \mathrm{C}$ for $10 \mathrm{~min}$. The PCR high fidelity system (Roche) was used. The DNA samples amplified with the fusion primers were purified with an Agencourt AMPure PCR Purification Kit according to the protocol procedure.

Amplicon library construction.-All PCR products from the nine brule obtained with the same pair of primers were pooled to constitute one sample. Another sample was obtained, pooling the PCR products from the corresponding samples outside. Four subsamples were obtained: IN (A-ITS1F/B-ITS2), OUT (A-ITS1F/B-ITS2), IN (A-ITS3/BITS4) and OUT (A-ITS3/B-ITS4). The quality of these samples was checked with the ND-1000 Spectrophotometer NanoDrop and the Experion ${ }^{\mathrm{TM}}$ System (Biorad), whereas the quantification was obtained with the ND-1000 Spectrophotometer NanoDrop. Two final amplicon libraries were built, one for inside and the other for outside, each containing fragments amplified with the two pairs of fusion primers. A total of $10 \mu \mathrm{L}$ containing $10^{10}$ molecules $/ \mu \mathrm{L}$ was necessary for each library. The concentration $(\mathrm{ng} / \mu \mathrm{L})$ of the samples to be mixed was converted into molecules $/ \mu \mathrm{L}$ with:

$$
\begin{aligned}
& \text { Molecules } / \mu \mathrm{L}= \\
& \frac{\text { Sample concentration }(\mathrm{ng} / \mu \mathrm{L}) \times\left(6.022 \times 10^{23}\right)}{\left(656.6 \times 10^{9}\right) \times \text { sample size }(\mathrm{bp})}
\end{aligned}
$$

The two libraries were pyrosequenced with Genome Sequencer FLX System (454 Life Science, Roche) by BMR 
Genomics s.r.l. (Padoa, Italy). The samples were processed in two lines out of the 16 available in the GS-FLX System.

Sequences analysis. - The four groups of sequences, two from inside the brule and two from outside, were separately analyzed. To reduce the overall error rate the reads containing one or more ambiguous nucleotides $(\mathrm{N})$ and reads shorter than 60 nucleotides were discarded (Sogin et al. 2006).

The primer sequence was trimmed with Sequencher. Each group of sequences (one for ITS- 1 from inside the brulé, one for ITS-1 from outside, one for ITS-2 from inside the brulé, one for ITS-2 from outside) was assembled into OTUs, defined for a $97 \%$ pairwise similarity, with the RapidOTU program available online (http://genome.jouy. inra.fr/rapidotu/). OTU richness was calculated by summing the number of OTUs, including singletons, within ITS-1 and ITS-2, inside and outside the brulé. Rarefaction curves and diversity indices, Ace and Chaol, also were calculated with the RapidOTU program. The non-default parameters used were alignment with Clustal W, distance Kimura 2 parameters and a cut-off of 0.03 . One representative sequence for each OTU was used for GenBank similarity queries (BLASTN on NCBI). A species level was assigned only when the e-value was 0.00 . When it was not possible to identify a clear similarity with a single-species sequence, the taxonomic rank assigned to each OTU was the genus that was in common with the first results of the top of the BLAST hits with a similar e-value. Results from BLASTN were organized in pie charts illustrating proportions for ITS1 from inside the brulé, for ITS-1 from outside, for ITS-2 from inside the brule, and for ITS2 from outside. Comparisons between ITS-1 and ITS-2 and between inside the brulé and outside were made through percentages. All the sequences grouped in the OTUs corresponding to $T$. melanosporum were brought into alignment separately to highlight any possible single nucleotide polymorphisms (SNPs).

When a polymorphism was identified a quality control for the corresponding base was done on the flow gram recovered for each sequence from the 454 platform to ensure that the polymorphism was correct. The sequences analyzed in this study were deposited in SRA under the accession number SRP006078.

\section{RESULTS}

Two pairs of primers, ITS1F/ITS2 and ITS3/ITS4, were used for the amplification of the ITS- 1 and the ITS-2 respectively from 18 DNA samples (nine from inside the brulé and nine from outside) and respectively produced fragments of about $350 \mathrm{bp}$ and $400 \mathrm{bp}$. Two libraries were generated, one for inside and the other for outside, each containing fragments amplified with the two pairs of primers. These libraries produced 15.788 reads; most of them were 200-300 bp long, with a consistent group of almost $300 \mathrm{bp}$. After the removal of low quality sequences (2142), 3568 and 3156 sequences were
TABlE I. Number of sequences obtained for each amplicon library (one for inside and the other for outside), before and after the removal of sequences of low quality

\begin{tabular}{lcc}
\hline \hline & Total sequences & Filtered sequences \\
\hline Inside brulé & & \\
ITS-1 $^{\text {a }}$ & 4101 & 3568 \\
ITS-2 $^{\mathrm{b}}$ & 3676 & 3156 \\
Outside brulé $_{\text {ITS-1 }}$ & \\
ITS-2 & 5160 & 4490 \\
\hline
\end{tabular}

${ }^{\text {a }}$ ITS-1 reads are obtained with the pair of primers A-ITS1F/B-ITS2.

${ }^{\mathrm{b}}$ ITS-2 reads are obtained with the pair of primers A-ITS3/ B-ITS4.

obtained from inside the brule with the ITS-1 and ITS-2 primers respectively. The sequences obtained from outside were 4490 with the ITS- 1 primers and 2432 with the ITS-2 primers (TABLE I).

All 8058 sequences of the ITS-1 region (from both inside and outside the brulé) were assembled to give a total of 900 OTUs; 213 were in common between the two habitats, 301 were exclusive to the inside brulé, while 386 were specific of the outside (FIG. 1A). Eight OTUs surpassed 100 sequences; 18 OTUs grouped from 50 to 100 sequences; 360 OTUs consisted of a single sequence (singletons). The singletons inside and outside were respectively 179 and 182 .

The 5588 sequences from the ITS-2 region were assembled into 885 OTUs. In this case 209 OTUs were in common to both inside and outside the brulé; 294 OTUs were exclusive of the outside, while 382 were exclusive of the inside (FIG. 1B). Altogether five OTUs surpassed 100 sequences, 12 OTUs grouped from 50 and 100 sequences and 375 OTUs consisted of a single sequence. The singletons inside and outside were respectively 218 and 161 .

BLAST queries indicated that most of the sequences obtained for both ITS fragments could be attributed to fungal organisms (FIG. 2). The nonfungal sequences amplified with the ITS-1 primers were 50 out of the 3568 inside and 151 out of the 4490 outside (1\% and $3 \%$ respectively) (FIG. 2). Among the remaining ITS-1 sequences only 163 sequences inside $(5 \%)$ and 175 outside (4\%) could not be classified to Phylum because they had low similarity with the specific fungal phyla and sometimes high similarity with sequences classified as uncultured fungi. With the pair of primers for the ITS-2 region, 181 of the 3156 sequences inside and 304 of the 2432 outside sequences ( $6 \%$ and $11 \%$ respectively) were not attributable to the fungal kingdom (FIG. 2). One hundred eighty-four sequences could not be assigned 

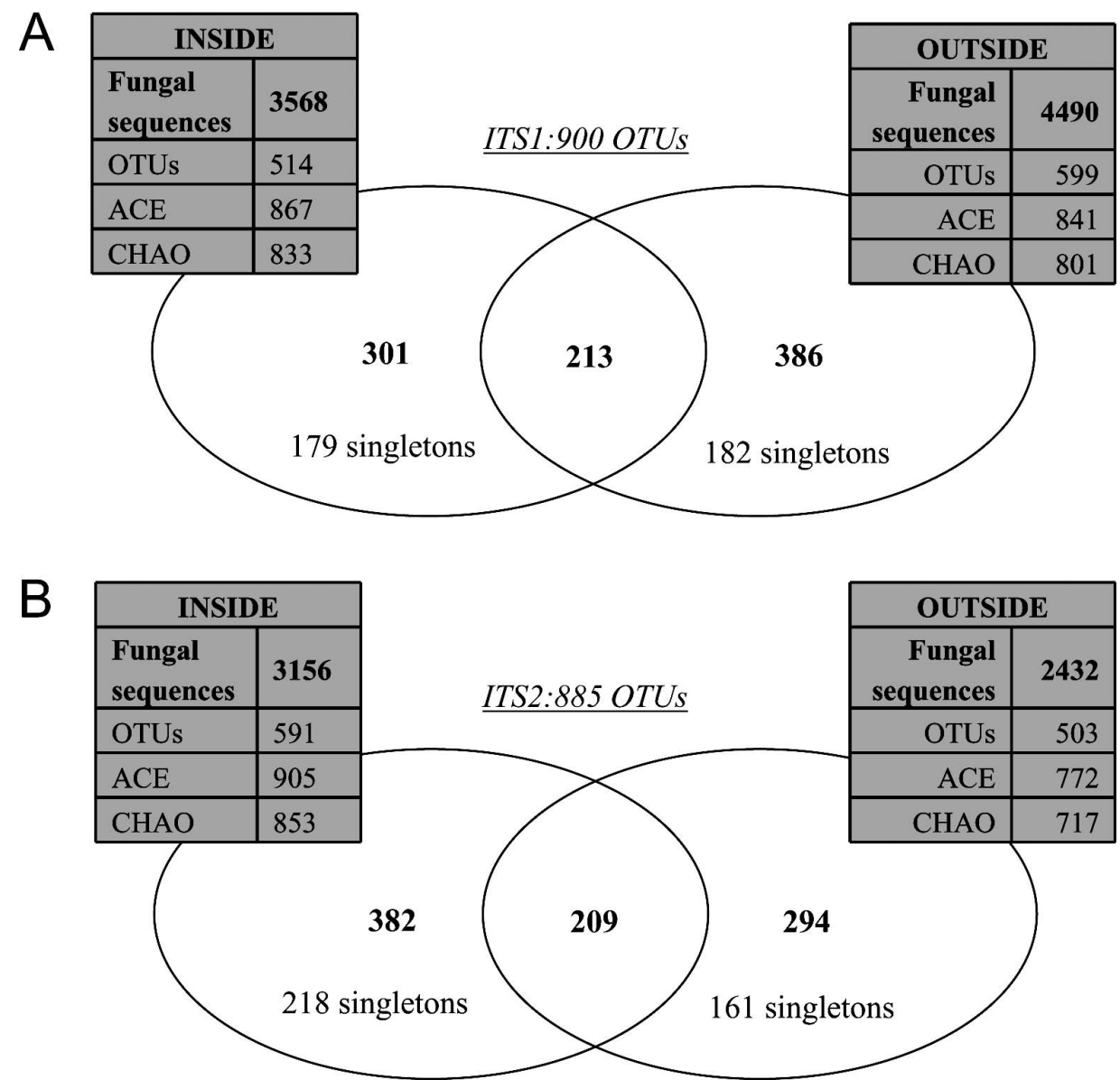

FIG. 1. Number of fungal sequences, operational taxonomic units (OTUs) and richness estimators Chaol and Ace from ITS-1 (A) and ITS-2 (B) are given for inside and outside the brulé. Venn diagram shows the overlap of the OTUs (defined at a similarity of $97 \%)$.

to any fungal phylum, 95 of which came from the inside library (3\%) and 79 from the outside $(3 \%)$.

Ascomycota and Basidiomycota were the dominant fungal phyla with a clear dominance of Ascomycota, which surpassed $50 \%$ of the sequences in all the four groups of sequences. Their number decreased, moving from inside to outside the brule with values of $72-57 \%$ (ITS-1) and 79-65\% (ITS-2). The number of basidiomycetes increased from $11 \%$ to $28 \%$ (ITS-1) and from $6 \%$ to $18 \%$ (ITS-2). Other phyla (Zygomycota, Chytridiomycota and Glomeromycota) were under $5 \%$, with the exception of Zygomycota which reached $8 \%$ inside the brule with the ITS-1 primers. Even though the number of sequences belonging to these phyla was low, the same trend was observed for the two pairs of primers; the number of Zygomycota and Chytridiomycota increased inside the brule, while the number of Glomeromycota decreased (FIG. 2).

The Sordariomycetes and Pezizomycetes were well represented in all four groups of sequences. The ITS-
1 reads revealed that $69 \%$ of the sequences outside and $78 \%$ inside belonged to the Sordariomycetes and Pezizomycetes. The greater number of sequences found inside was due mostly to an increase in Pezizomycetes, which rose from $31 \%$ to $45 \%$ (SuPPLEMENTAL FIG. 1).

The class composition changed with the ITS-2 reads. A greater percentage of Dothideomycetes and Eurotiomycetes was found in comparison with the ITS-1 reads. In other words, the number of Sordariomycetes increased from $18 \%$ to $40 \%$ while the number of Eurotiomycetes decreased from $26 \%$ to $4 \%$, moving from outside to inside the brulé (SuPPleMENTAL FIG. 2).

The most representative orders in three out of the four groups of sequences were Hypocreales and Pezizales. The fourth group from outside the brule ITS-2 also presented in addition to a low percentage of Hypocreales $(14 \%)$ a high percentage of the Onygenales $(21 \%)$ and was not represented in the ITS-1 reads (SUPPLEMENTAL FIG. 3). 

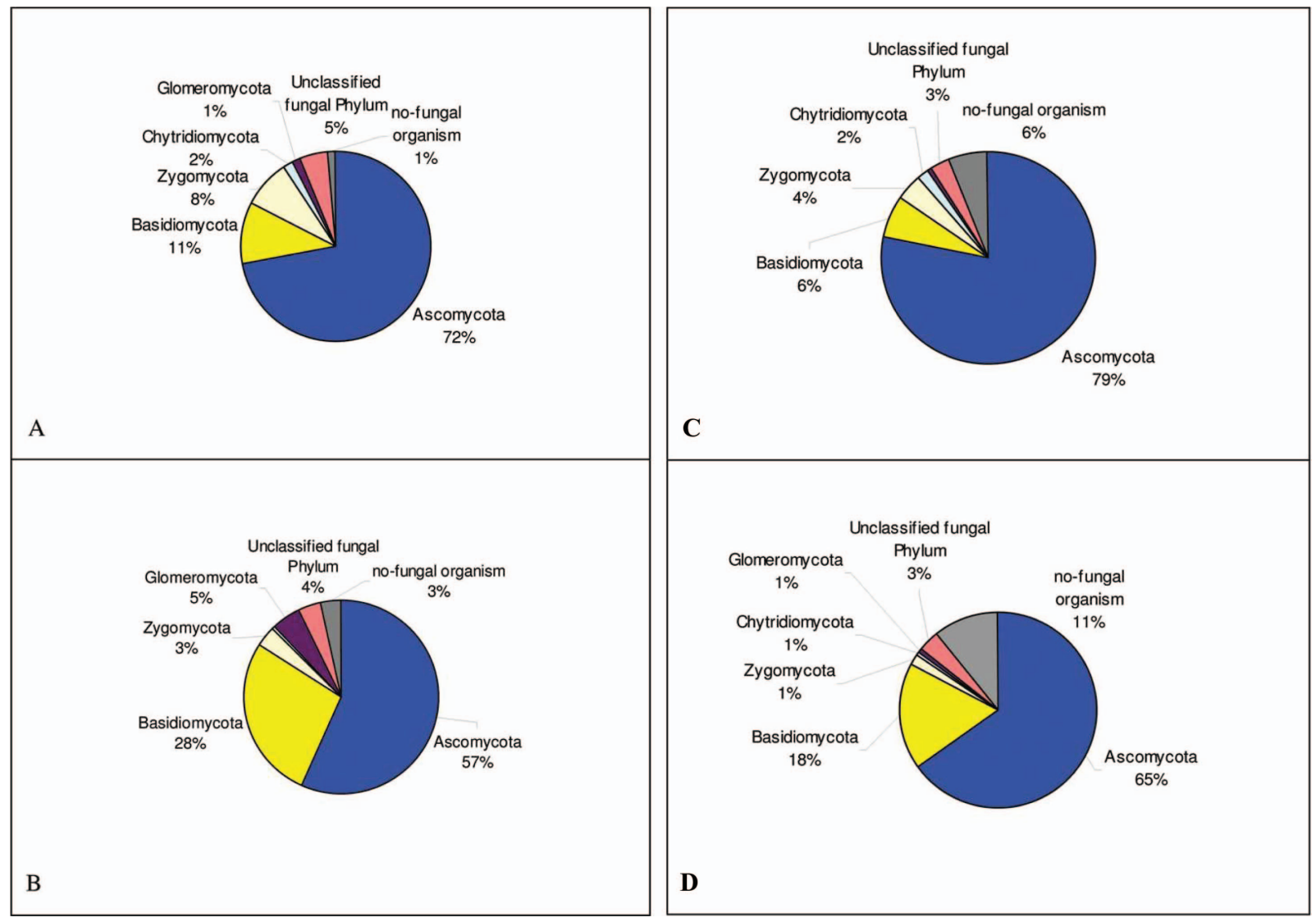

FIG. 2. Phyla subdivision from: ITS-1 reads for inside (A) and outside (B) the brulé; ITS-2 reads for inside (C) and outside (D) the brulé.

Among the Basidiomycota more than $80 \%$ of the sequences from ITS-1 and ITS-2 represented two classes, Agaricomycetes and Tremellomycetes. Moving from inside the brule to outside, the Agaricomycetes increased from $43 \%$ to $80 \%$ (ITS-1) and from $55 \%$ to $82 \%$ (ITS-2), while the Tremellomycetes decreased from $43 \%$ to $8 \%$ (ITS-1) and from $33 \%$ to $13 \%$ (ITS-2) (SUPPlemental FigS. 4, 5). A higher taxa subdivision proved to be difficult because of the abundance of unclassified sequences. In all four groups of sequences more than $10 \%$ of those belonging to Basidiomycota were not classified in any order. For the outside ITS-1 sequences the percentage of unclassified sequences reached 39\%. Moreover the results showed that the most highly represented orders among the Agaricomycetes were Agaricales and Thelephorales, while among the Tremellomycetes it was the Filobasidiales (SupPlemental Fig. 6). Comparisons of relative abundances of classes and orders are illustrated (SuPPLEMENTAL FIGS. 1-6) and summarized (TABLE II).

Eight out of 900 ITS-1 OTUs from both inside and outside contained more than 100 sequences. Among these a single OTU comprised a total of 1305 sequences, 848 from inside and 457 from outside the brulé. BLAST queries returned a $100 \%$ similarity with GenBank T. melanosporum sequences for this OTU. The sequences grouped into this OTU were searched for the presence of SNPs and carefully divided into subgroups. With the exception of one subgroup that contained reads that occurred only once in the entire dataset, which therefore was excluded from this analysis because of possible sequencing mistakes, 16 subgroups differing for 1, 2 or more SNPs were identified. Three subgroups were the most abundant.

Another three abundant OTUs, assigned to Ascomycota, were classified into the Cordyceps, Genea and Bionectria genera. Sequences belonging to Genea, which is an ectomycorrhizal genus, were not found inside the brulé but were found outside (198 sequences). The OTUs assigned to Basidiomycota belonged to the genera Hymenogaster and Cryptococcus. While the Hymenogaster sequences were numerous outside the brulé, Cryptococcus was found mainly inside (TABLE III).

Four OTUs from ITS-2 belonged to Ascomycota and contained more than 100 sequences. Again in this case we found a close correspondence with the genera Tuber, Cordyceps and Bionectria, already found with the ITS-1 reads. The number of sequences for 
TABLE II. BLAST results of OTUs from ITS-1 and ITS-2 both inside and outside the brulé

\begin{tabular}{|c|c|c|c|c|c|c|c|c|c|}
\hline \multicolumn{2}{|c|}{ Ascomycota ITS1 } & \multicolumn{2}{|c|}{ Ascomycota ITS2 } & \multirow{2}{*}{$\frac{\text { Ascomycota }}{\text { Class }}$} & \multirow{2}{*}{$\frac{\text { Basidiomycota }}{\text { Class }}$} & \multicolumn{2}{|c|}{ Basidiomycota ITS1 } & \multicolumn{2}{|c|}{ Basidiomycota ITS2 } \\
\hline Inside & Outside & Inside & Outside & & & Inside & Outside & Inside & Outside \\
\hline $33 \%{ }^{\mathrm{a}}$ & $38 \%$ & $40 \%$ & $18 \%$ & Sordariomycetes & Agaricomycetes & $43 \%$ & $80 \%$ & $55 \%$ & $82 \%$ \\
\hline $45 \%$ & $31 \%$ & $20 \%$ & $16 \%$ & Pezizomycetes & Tremellomycetes & $43 \%$ & $8 \%$ & $33 \%$ & $13 \%$ \\
\hline $1 \%$ & $1 \%$ & $4 \%$ & $26 \%$ & Eurotiomycetes & Pucciniomycetes & - & $2 \%$ & $6 \%$ & $2 \%$ \\
\hline $8 \%$ & $8 \%$ & $14 \%$ & $18 \%$ & Dothideomycetes & Microbotryomycetes & $3 \%$ & $1 \%$ & $3 \%$ & - \\
\hline $3 \%$ & $4 \%$ & $5 \%$ & $10 \%$ & Leotiomycetes & Cystobasidiomycetes & $1 \%$ & - & - & - \\
\hline- & $1 \%$ & - & $1 \%$ & Orbiliomycetes & Unclassified & $10 \%$ & $9 \%$ & $3 \%$ & $3 \%$ \\
\hline- & $1 \%$ & $1 \%$ & $1 \%$ & Lecanoromycetes & & & & & \\
\hline- & $2 \%$ & - & - & Saccaromycetes & & & & & \\
\hline \multirow[t]{17}{*}{$10 \%$} & $14 \%$ & $16 \%$ & $10 \%$ & Unclassified & & & & & \\
\hline & & Inside & Outside & Order & Order & Inside & Outside & & \\
\hline & & $20 \%$ & $16 \%$ & Pezizales & Agaricales & $7 \%$ & $37 \%$ & & \\
\hline & & $2 \%$ & $21 \%$ & Onygenales & Polyporales & $1 \%$ & - & & \\
\hline & & $1 \%$ & $5 \%$ & Chaetothyriales & Filobasidiales & $33 \%$ & $3 \%$ & & \\
\hline & & $8 \%$ & $9 \%$ & Pleosporales & Sporidiobolales & $3 \%$ & - & & \\
\hline & & $2 \%$ & $3 \%$ & Capnodiales & Cantharellales & - & $2 \%$ & & \\
\hline & & $3 \%$ & $2 \%$ & Sordariales & Pucciniales & - & $1 \%$ & & \\
\hline & & $4 \%$ & $5 \%$ & Helotiales & Boletales & $13 \%$ & $2 \%$ & & \\
\hline & & - & $1 \%$ & Myriangiales & Tremellales & $8 \%$ & $3 \%$ & & \\
\hline & & $2 \%$ & $1 \%$ & Xylariales & Russulales & - & $1 \%$ & & \\
\hline & & $1 \%$ & $1 \%$ & Lecanorales & Telephorales & $16 \%$ & $12 \%$ & & \\
\hline & & - & $1 \%$ & Botryosphaeriales & Unclassified & $19 \%$ & $39 \%$ & & \\
\hline & & - & $1 \%$ & Orbiliales & & & & & \\
\hline & & $1 \%$ & - & Verrucariales & & & & & \\
\hline & & $34 \%$ & $14 \%$ & Hypocreales & & & & & \\
\hline & & $22 \%$ & $21 \%$ & Unclassified & & & & & \\
\hline
\end{tabular}

${ }^{\text {a }}$ Percentages refer to the number of sequences after selection obtained from ITS-1, 3568 inside and 4490 outside.

inside and outside the brulé changed for each OTU (TABLE IV).

Rarefaction curves generated within ITS-1 and ITS2 , inside as well as outside the brulé, did not reach saturation, indicating that the number of sequences obtained for each habitat did not capture the real richness of soil samples in ITS-1 or in ITS-2 (FIG. 3). The Chao 1 and Ace non-parametric richness estimators revealed that our results were far from the expected richness of the soil sample (FIG. 1).

\section{DISCUSSION}

Napoli et al. (2010) used DGGE to examine differences between fungal populations sampled inside and outside brulé areas of nine $T$. melanosporum/Q. pubescens truffle grounds. Because the aim of the present investigation was to see how the short reads from ITS-1 and ITS-2 differed in the sequence and OTU numbers and in the taxa assignment we took the advantage of the extensive sampling previously carried out for DGGE and of our knowledge of truffle ground fungal populations to validate pyrose- quencing. This new technique enables extraordinary progress in assessing biodiversity and contributes to our understanding of the microbial diversity in ecosystems.

Most of the sequences obtained by pyrosequencing were grouped in a few principal OTUs. Although an easier amplification of particular groups could be due to a better DNA accessibility or to primer bias, the results make it possible to hypothesize that these particular groups could be the main fungal taxa present in the sampled soil. Members of the Ascomycota were recovered as the dominant group in our investigation with both ITS regions. Some principal OTUs were assigned for both regions to identical taxa, in particular to Cordyceps, Tuber and Bionectria. Other sequences were not common to the two regions, at least in the principal OTUs, but a clear tendency was identified. Fewer ascomycete sequences were recovered from outside the brulé, while the number of Basidiomycota sequences increased. Within subdivisions of the Basidiomycota and Ascomycota we noted that this trend was due mainly to changes in abundance of members of some principal orders. 


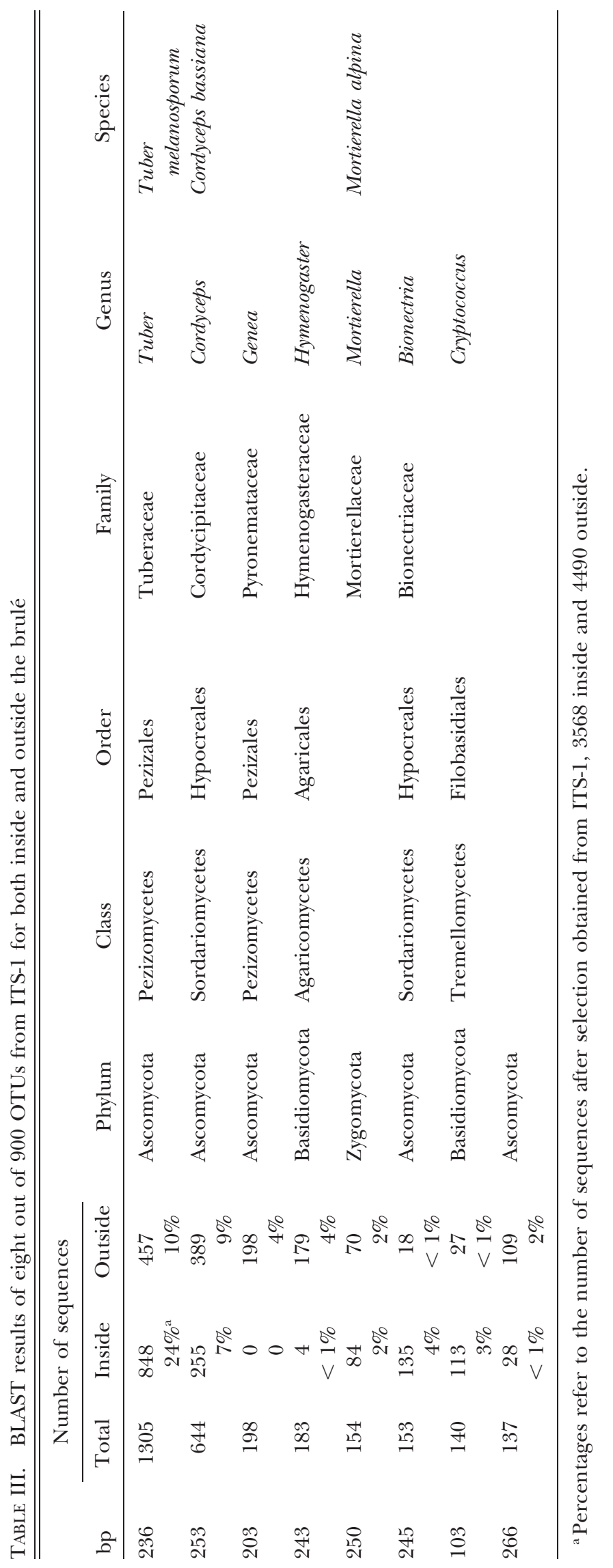


TABLE IV. BLAST results of four out of 885 OTUs from ITS-2 both inside and outside the brulé

\begin{tabular}{|c|c|c|c|c|c|c|c|c|c|}
\hline \multirow[b]{2}{*}{ bp } & \multicolumn{3}{|c|}{ Number of sequences } & \multirow[b]{2}{*}{ Phylum } & \multirow[b]{2}{*}{ Class } & \multirow[b]{2}{*}{ Order } & \multirow[b]{2}{*}{ Family } & \multirow[b]{2}{*}{ Genus } & \multirow[b]{2}{*}{ Species } \\
\hline & Total & Inside & Outside & & & & & & \\
\hline 197 & 569 & $\begin{array}{l}433 \\
14 \% \mathrm{a}\end{array}$ & $\begin{array}{l}136 \\
6 \%\end{array}$ & Ascomycota & Sordariomycetes & Hypocreales & Cordycipitaceae & Cordyceps & $\begin{array}{c}\text { Cordyceps } \\
\text { bassiana }\end{array}$ \\
\hline 89 & 345 & $\begin{array}{l}222 \\
7 \%\end{array}$ & $\begin{array}{l}123 \\
5 \%\end{array}$ & Ascomycota & Pezizomycetes & Pezizales & Tuberaceae & Tuber & \\
\hline 225 & 173 & $\begin{array}{l}160 \\
5 \%\end{array}$ & $\begin{array}{l}13 \\
<1 \%\end{array}$ & Ascomycota & Sordariomycetes & Hypocreales & Bionectriaceae & Bionectria & \\
\hline 60 & 104 & $\begin{array}{l}86 \\
3 \%\end{array}$ & $\begin{array}{l}18 \\
<1 \%\end{array}$ & Ascomycota & & & & & \\
\hline
\end{tabular}

${ }^{a}$ Percentages refer to the number of sequences after selection obtained from ITS-2, 3156 inside and 2432 outside.

Among the Ascomycota the number of Hypocreales increased inside the brulé. Among the Basidiomycota the number of Agaricales increased while the number of Filobasidiales decreased outside the brulé. It is known that the Hypocreales and Filobasidiales mainly include saprotrophic fungi, while many of the common ectomycorrhizal fungi belong to the Agaricales. On the basis of these data, we suggest a reduction in the number of ectomycorrhizal species within the brulé.

The OTU assigned to the genus Tuber was an exception. Although this genus contains ectomycorrhizal fungi, it was found mainly inside the brulé. Species determination for this principal OTU was possible only with one of the two regions. In fact with the ITS-1 region it was possible to assign this principal OTU to T. melanosporum. This result agrees with data by Napoli et al. (2010) who suggested a competitive effect of T. melanosporum with the other ectomycorrhizal fungi. With the ITS-2 region it was not possible to clearly identify the Tuber species, principally because the length of the sequences was less than $90 \mathrm{bp}$. In an attempt to remove all sequences shorter than $200 \mathrm{bp}$ to improve the match in the BLAST analysis, this OTU was dropped. This prevented us from discarding reads shorter than $200 \mathrm{bp}$ at the beginning of our analysis.

Searching for SNPs, the three most highly represented subgroups of $T$. melanosporum likely belong to the ubiquitous haplotypes 1,2 or 3 described in fruiting bodies by Murat et al. (2004) and Riccioni et al. (2008); not one subgroup matched the potentially new haplotypes described by Napoli et al. (2010) in soil, whereas five potentially new haplotypes have been identified. This suggests the existence of a greater variability of $T$. melanosporum in the soil than has been recognized in the fruiting bodies, as reported by Zampieri et al. (2010) who found a new haplotype in the $\beta$-tubulin gene of $T$. magnatum mycelium in soil. Because a higher variability usually increases the chance of adaptability and fitness of the mycelia this could ensure mycelia suitable for fruiting body production and must be considered in management of plantations (Zampieri et al. 2010).

PCR biases in ITS-1 and ITS-2.-It is known that various commonly used ITS primers might introduce biases during the amplification of different parts of the ITS region. The pair of primers ITS1-F/ITS2 here used for the amplification of the ITS-1 region produced fewer non-fungal sequences in addition to a higher number of sequences than the pair ITS3/ITS4 amplifying the ITS-2 region. In fact our work produced 15788 reads among which 12960 were fungal sequences; $99 \%$ (inside) and $97 \%$ (outside) originated from ITS-1, whereas 94\% (inside) and 88\% (outside) came from ITS-2. Given that ITS1-F is a fungal specific primer, it is not surprising to find more fungal sequences in the ITS-1 region data. As a consequence, the pair of primers ITS1-F/ITS2 was more selective. The long fragments inhibit the emulsion-PCR step at the expense of the short amplicons, according with Margulies et al. (2005). In this view the longer fragment of the ITS-2 probably inhibited the emulsion-PCR step at the expense of the shorter fragment of the ITS-1, and this could be the reason for the lower number of sequences obtained from the ITS-2.

In silico PCR analyses conducted by Bellemain et al. (2010) indicated that ITS3 and ITS4 primers preferentially amplified ascomycetes whereas ITS1-F preferentially amplified basidiomycetes. In agreement with these authors, we have found, using the ITS-2 region, a slighter higher number of the ascomycetes compared to basidiomycetes in both environments.

In term of species richness the ITS- 1 and ITS-2 pyrosequencing produced a high richness, 900 OTUs and 885 OTUs respectively. The OTU number inside the brule was $514(14 \%)$ and $591(19 \%)$ on the basis of ITS-1 and ITS-2 respectively, against 599 (13\%) and $503(21 \%)$ outside. Nevertheless, if we consider the high number of singletons found in ITS- 1 and in ITS- 


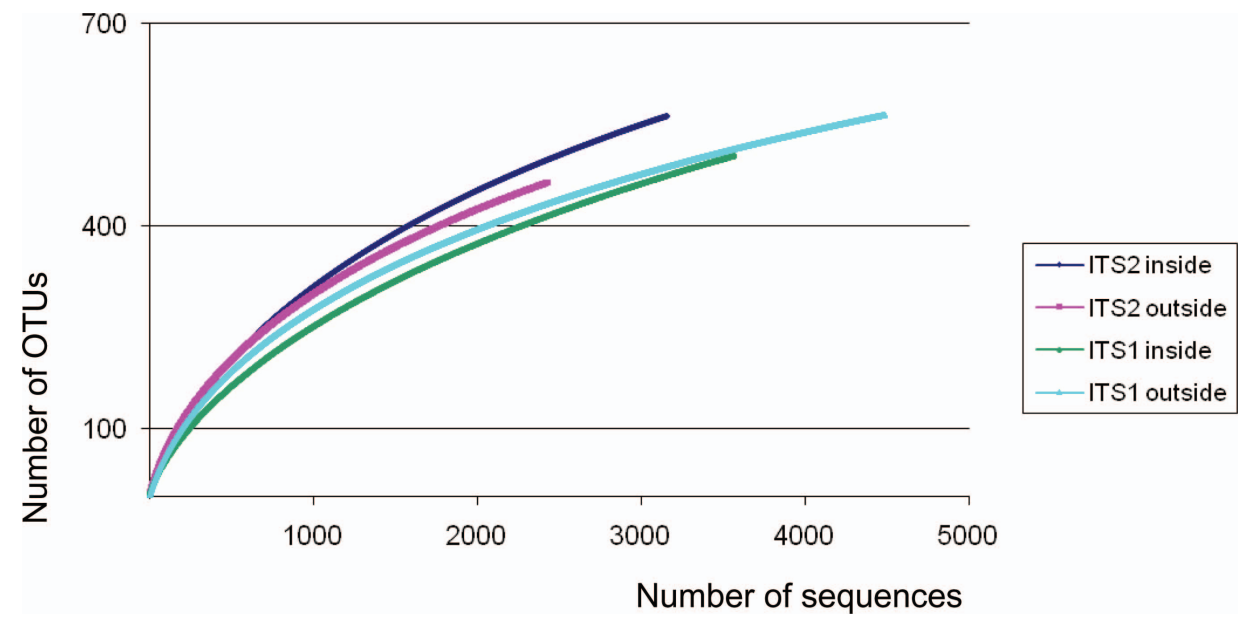

FIG. 3. Rarefaction curves generated by the DOTUR 1.53 software for the fungal operational taxonomic units (OTUs) obtained from ITS-1 and ITS-2 for inside as well as outside the brulé.

2, the richness estimation might not be reliable. This conclusion is supported by Jumpponen and Jones (2009) who found one-half of singletons in the hyperdiverse phyllosphere communities. Regardless of the high richness, the rarefaction curves did not reach saturation in either ITS-1 or ITS-2, suggesting that the real fungal richness could be greater. Furthermore the numerous non-fungal sequences revealed by the ITS- 2 region could overwhelm the number of obtained fungal OTUs and consequently affect the slope of the rarefaction curve for this region. In agreement with the analysis by Nilsson et al. (2009), who examined how well the short pyrosequenced reads perform in silico in taxa assignment, we have demonstrated that both ITS-1 and ITS-2 provide adequate information for taxon assignment. Both regions led to the knowledge we gained on fungal populations in one of the previously investigated truffle grounds (i.e. ectomycorrhizal species decreased inside the brulé, but only ITS-1 revealed that the ectomycorrhizal fungus $T$. melanosporum was mainly inside the brulé).

To the authors' knowledge this is the first report on the comparison of the two ITS regions in pyrosequencing. Buée et al. (2009), Jumpponen and Jones (2009) and Jumpponen et al. (2010) used ITS-1 to study soil forest fungi, fungal communities in the Quercus macrocarpa phyllosphere and fungal communities in the Quercus spp. respectively. Tedersoo et al. (2010) showed on the basis of the ITS-1 sequence data that pyrosequencing and Sanger sequencing of tropical fungi provide similar results but reveal substantial methodological biases. Apart from the ITS region, Lumini et al. (2010) and Opik et al. (2009) used the small subunit ribosomal RNA gene to address arbuscular mycorrhizal fungi biodiversity. On the basis of our experience, ITS-1 is probably the best choice to study soil forest fungi. Because our pilot experiment of comparison of the short ITS-1 and ITS-2 took advantage of our previous knowledge on truffle ground fungal populations we did not include repetitions and consequently statistical analysis. Nevertheless we suggest considering repetitions when taxa have to be inferred, once the molecular marker has been chosen.

Taken together, we have provided an experimental validation of the efficacy of the short ITS-1 and ITS-2 when applied to 454 pyrosequencing. In times in which high throughput techniques are becoming widely used, bypassing DNA cloning, validation experiments represent an important point from which to build next steps. Thanks to this technology the scientific challenge is a sequencing project to uncover the soil metagenome (Vogel et al. 2009). This surely will unravel the biodiversity associated with soil microorganisms, in addition to providing insights into the ecology of those that are beneficial to crop production.

\section{ACKNOWLEDGMENTS}

This research was financed by the Compagnia di San Paolo to PB (IPP-CNR) and by Regione Piemonte to PB and AM (IPP-CNR). We thank P. Sourzat from Station d'experimentation sur la truffe, Le Montat, France, for granting us access to the truffle grounds. We especially thank M. Girlanda for the bioinformatic advice and A. Vizzini for helping in the database interpretation.

\section{LITERATURE CITED}

Bellemain E, Carlsen T, Brochmann C, Coissac E, Taberlet P, Kauserud H. 2010. ITS as an environmental DNA barcode for fungi: an in silico approach reveals 
potential PCR biases. BMC Microbiol 10:189, doi:10.1186/1471-2180-10-189

Buée M, Reich M, Murat C, Morin E, Nilsson RH, Uroz S, Martin F. 2009. 454 Pyrosequencing analyses of forest soils reveal an unexpectedly high fungal diversity. New Phytol 184:449-456, doi:10.1111/j.1469-8137.2009. 03003.x

Horton TR, Bruns TD. 2001. The molecular revolution in ectomycorrhizal ecology: peeking into the black box. Mol Ecol 10:1855-1871, doi:10.1046/j.0962-1083. 2001.01333.x

Jumpponen A, Jones KL. 2009. Massively parallel 454 sequencing indicates hyperdiverse fungal communities in temperate Quercus macrocarpa phyllosphere. New Phytol 18:438-448, doi:10.1111/j.1469-8137.2009.02990.x

- - Mattox D, Yaege C. 2010. Massively parallel 454 sequencing of fungal communities in Quercus spp. ectomycorrhizas indicates seasonal dynamics in urban and rural sites. Mol Ecol 19:41-53, doi:10.1111/ j.1365-294X.2009.04483.x

Lilleskov EA, Parrent JL. 2007. Can we develop general predictive models of mycorrhizal fungal communityenvironment relationships? New Phytol 174:250-256, doi:10.1111/j.1469-8137.2007.02023.x

Luis P, Walther G, Kellner H, Martin F, Buscot F. 2004. Diversity of laccase genes from basidiomycetes in a forest soil. Soil Biol Biochem 36:1025-1036, doi:10.1016/j.soilbio.2004.02.017

Lumini E, Orgiazzi A, Borriello R, Bonfante P, Bianciotto V. 2010. Disclosing arbuscular mycorrhizal fungal biodiversity in soil through a land-use gradient using a pyrosequencing approach. Environ Microbiol 12:21652179.

Margulies M, Egholm M, Altman WE, Attiya S, Bader JS, Bemben LA, et al. 2005. Genome sequencing in microfabricated high-density picolitre reactors. Nature 437:376-380.

Mello A, Murat C, Bonfante P. 2006. Truffles: much more than a prized and local fungal delicacy. FEMS Microbiol Lett 260:1-8, doi:10.1111/j.1574-6968.2006.00252.x

Murat C, Diez J, Luis P, Delaruelle C, Dupré C, Chevalier G, Bonfante P, Martin F. 2004. Polymorphism at the ribosomal DNA ITS and its relation to postglacial recolonization routes of the Perigord truffle Tuber melanosporum. New Phytol 164:401-411, doi:10.1111/ j.1469-8137.2004.01189.x

Napoli C, Mello A, Borra A, Vizzini A, Sourzat P, Bonfante P. 2010. Tuber melanosporum, when dominant, affects fungal dynamics in truffle grounds. New Phytol 185: 237-247, doi:10.1111/j.1469-8137.2009.03053.x

Nilsson RH, Ryberg M, Abarenkov K, Sjökvist E, Kristiansson E. 2009. The ITS region as a target for characterization of fungal communities using emerging sequencing technologies. FEMS Microbiol Lett 296:97-101, doi:10.1111/j.1574-6968.2009.01618.x
O’Brien HE, Parrent JL, Jackson JA, Moncalvo JM, Vilgalys R. 2005. Fungal community analysis by large scale sequencing of environmental samples. Appl Environ Microbiol 71:5544-5550, doi:10.1128/AEM.71.9. 5544-5550.2005

Opik M, Metsis M, Daniell TJ, Zobel M, Moora M. 2009. Large scale parallel 454 sequencing reveals host ecological group specificity of arbuscular mycorrhizal fungi in a boreonemoral forest. New Phytol 184:424437, doi:10.1111/j.1469-8137.2009.02920.x

Reich M, Kohler A, Martin F, Buée M. 2009. Development and validation of an oligonucleotide microarray to characterize ectomycorrhizal fungal communities. BMC Microbiol 9:241, doi:10.1186/1471-2180-9-241

Riccioni C, Belfiori B, Rubini A, Passeri V, Arcioni S, Paolocci F. 2008. Tuber melanosporum outcrosses: analysis of the genetic diversity within and among its natural populations under this new scenario. New Phytol 180:466-478, doi:10.1111/j.1469-8137.2008. 02560.x

Roesch LFW, Furlthorpe RR, Riva A, Casella G, Hadwin AKM, Kent AD, et al. 2007. Pyrosequencing enumerates and contrasts soil microbial diversity. ISME J 1:283-29.

Ronaghi M. 2001. Pyrosequencing sheds light on DNA sequencing. Genome Res 11:3-11, doi:10.1101/ gr.11.1.3

— Uhlén M, Nyrén P. 1998. A sequencing method based on real-time pyrophosphate. Science 17:363-365, doi:10.1126/science.281.5375.363

Sogin ML, Morrison HG, Huber JA, Mark Welch D, Huse SM. 2006. Microbial diversity in the deep sea and the underexplored "rare biosphere". PNAS 103:1211512120, doi:10.1073/pnas.0605127103

Suz LM, Martin MP, Oliach D, Fischer RC, Colinas C. 2008. Mycelial abundance and other factors related to truffle productivity in Tuber melanosporum-Quercus ilex orchards. FEMS Microbiol Lett 285:72-78, doi:10.1111/ j.1574-6968.2008.01213.x

Tedersoo L, Nilsson RH, Abarenkov K, Jairus T, Sadam A, Saar I, Bahram M, et al. 2010. 454 pyrosequencing and Sanger sequencing of tropical mycorrhizal fungi provide similar results but reveal substantial methodological biases. New Phytol 188:291-301, doi:10.1111/ j.1469-8137.2010.03373.x

Vogel TM, Simonet P, Jansson J, Hirsch PR, Tiedje JM, van Elsas JD, Bailey MJ, Nalin R, Philippot L. 2009. TerraGenome: a consortium for the sequencing of a soil metagenome. Nat Rev Microbiol 7:252, doi: 10.1038/nrmicro2119

Zampieri E, Murat C, Cagnasso M, Bonfante P, Mello A. 2010. Soil analysis reveals the presence of an extended mycelial network in a Tuber magnatum truffle ground. FEMS Microbiol Ecol 71:43-49, doi:10.1111/ j.1574-6941.2009.00783.x 